\title{
Bearing Fault Signal Analysis Based on an Adaptive Multiscale Combined Morphological Filter
}

\author{
Chun Lv $\mathbb{D}^{1}{ }^{1}$ Peilin Zhang, ${ }^{1}$ Dinghai $W u,{ }^{1}$ Bing Li $\mathbb{D}^{2},{ }^{2}$ and Yunqiang Zhang ${ }^{1}$ \\ ${ }^{1}$ Department of Vehicle and Electrical Engineering, Army Engineering University, Shijiazhuang 050003, China \\ ${ }^{2}$ Department of Electronic Information Engineering, Shantou University, Shantou 515063, China \\ Correspondence should be addressed to Chun Lv; lvchun18@163.com
}

Received 28 October 2019; Revised 30 January 2020; Accepted 18 February 2020; Published 3 March 2020

Academic Editor: Hyeong Joon Ahn

Copyright $(2020$ Chun Lv et al. This is an open access article distributed under the Creative Commons Attribution License, which permits unrestricted use, distribution, and reproduction in any medium, provided the original work is properly cited.

\begin{abstract}
Bearing fault signal analysis is an important means of bearing fault diagnosis. To effectively eliminate noise in a fault signal, an adaptive multiscale combined morphological filter is proposed based on the theory of mathematical morphology. Both simulation and experimental results show that the adaptive multiscale combined morphological filter can remove noise more thoroughly and retain details of the fault signal better than the dual-tree complex wavelet filter, traditional morphological filter, adaptive singular value decomposition method (ASVD), and improved switching Kalman filter (ISKF). The adaptive multiscale combined morphological filter considers both positive and negative impulses in the signal; therefore, it has strong adaptability to complex noise in the environment, making it an effective new method for bearing fault diagnosis.
\end{abstract}

\section{Introduction}

Bearings are important components in rotating mechanical equipment, and their operating condition affects the overall working state of mechanical equipment. A bearing is prone to defects after prolonged use, and these defects can be useful during bearing fault diagnosis [1-4]. When a bearing fails, its vibration signal is composed of a modulation signal, periodic pulse signal, and noise signal. The periodic pulse signal is caused by the bearing fault, and the bearing fault type can be determined by analyzing the characteristic frequency of the periodic pulse signal. Due to the complex working environment of the bearing, the vibration signals of the bearing collected by relevant equipment show obvious nonstationary and nonlinear characteristics [5-9].

The mathematical morphology filter, developed on the basis of mathematical morphology transformation, is an effective method of analyzing nonlinear signals. The mathematical morphology filter selects appropriate structuring elements to decompose the signal into several components, according to the geometric characteristics of the signal. Even if there is strong noise or distortion in the signal, the basic morphological characteristics of the signal can be preserved after the signal is filtered. Compared with other signal analysis methods, the mathematical morphology filter, which has been widely used in pattern recognition, image processing, computer vision, power signals, ECG and EEG signal processing, mechanical equipment fault diagnosis, and other fields, has the advantages of easy implementation, fast algorithms, and minimal calculations [10-14]. Because the collected vibration signal of the bearing inevitably contains noise, the frequencies of the noise and fault signal will overlap in the frequency spectrum. A traditional morphological filter uses a single-scale operation, so its noise-filtering effects are general; in addition, it may filter out useful fault signals, which may prevent a true reflection of the bearing fault signals [15-18].

An adaptive multiscale combined morphological filter is developed based on the concept of multiscale overall filtering to overcome the shortcomings of the traditional morphological filter. Both simulation and experimental results show that the adaptive multiscale combined morphological filter has a better denoising effect and can retain useful signals better than the traditional filter. 


\section{Summary of Mathematical Morphology Filtering Theory}

2.1. Basic Morphology Operators. Dilation and erosion are the two basic operators of mathematical morphology. The collected vibration signal of the bearing is a one-dimensional dispersed signal, so this paper only introduces a one-dimensional dispersed gray-value morphological transformation.

$f(n)$ is defined as a disperse one-dimensional input signal, and its domain is $F=\{0,1,2 \cdots, N-1\} . g(m)$ is the structuring element, and its domain is $G=\{0,1,2 \cdots, M-1$ \}; in addition, $M \ll N$.

The dilation of $f(n)$ on $g(m)$ is

$$
(f \oplus g)(n)=\max \{f(n-m)+g(m)\} .
$$

The erosion of $f(n)$ on $g(m)$ is

$$
(f \Theta g)(n)=\min _{m \in G}\{f(n+m)-g(m)\} .
$$

The dilation operation is equivalent to the maximum value filtering of the disperse function in the sliding filter window (structuring element), which widens the peak value as well as increases the valley value of the signal. The erosion operation is equivalent to the minimum value filtering of the disperse function in the sliding filter window (structuring element), which widens the valley value as well as increases the peak value of the signal. Dilation and erosion, as two basic operations, can be used to form opening operations and closing operations, and their expressions are as follows:

The opening operation

$$
(f \circ g)(n)=(f \Theta g \oplus g)(n) .
$$

The closing operation

$$
(f \cdot g)(n)=(f \oplus g \Theta g)(n) .
$$

From Equation (3) and Equation (4), it can be seen that the opening operation means the signal is eroded first and then dilated by the structuring element, and the closing operation means the signal is dilated first and then eroded by the structuring element. The opening operation can eliminate the isolated point and weaken the positive pulse interference in the signal to smoothen the signal waveform. The closing operation can repair the holes and gaps, fill the grooves to make them flat, and suppress the negative pulse interference in the signal waveform.

Selection of the structuring element used in morphological transformation is also very important because different structuring elements have different effects on signal processing. Several common types of structuring elements are flat, circular, cosine, triangular, and curved. The flat structuring element has the following advantages: the amplitude is zero, the calculation is simple and fast, the signal processing efficiency is high, and the processing effect can be guaranteed. Therefore, in this paper, a flat structuring element is selected for the morphological filtering of the bearing fault signal.
2.2. Traditional Morphological Filter. Because the dilation, erosion, opening, and closing operations can only filter one of the positive and negative impulse noise in the signal, in many cases, the opening and closing operations are combined to construct the morphological closing-opening filter and morphological opening-closing filter, which are as shown in Equation (5) and Equation (6), respectively.

$$
\begin{aligned}
& F_{C O}(f(n))=(f \cdot g \circ g)(n) . \\
& F_{O C}(f(n))=(f \circ g \cdot g)(n) .
\end{aligned}
$$

Morphological closing-opening and opening-closing filters combine two kinds of operations (opening and closing), which can weaken both positive and negative impulse noise in the signal, but they cannot avoid the statistical bias of the filtering results caused by the dilatability of the closing operation and the erodibility of the opening operation. To achieve a better filtering effect, the combined morphological filter is usually constructed by combining morphological closing-opening and opening-closing filters, as shown in Equation (7).

$$
F_{C O-O C}(f(n))=\frac{1}{2}\left[F_{C O}(f(n))+F_{O C}(f(n))\right] .
$$

2.3. Multiscale Morphological Filter. Originally, multiscale morphology was established to enrich the practicability of morphology in the field of image processing and shorten the operation time when the size of the structuring element is large, which is based on decomposing morphological structuring elements. When applied to the research of a onedimensional vibration signal, the structuring element of the multiscale morphological filter can be obtained by corresponding time dilations of the unit structuring element itself.

If the structuring element of scale $i$ is $i g$ and the unit structuring element is $g$, then ig can be obtained by $i-1$ times dilation of $g$ itself, as shown in Equation (8).

$$
i g=\underbrace{g \oplus g \oplus \cdots \oplus g}_{i-1 \text { times }} .
$$

The dilation and erosion operations of signal $f$ by multiscale structuring elements are as shown in Equation (9) and Equation (10), respectively:

$$
\begin{aligned}
& f \oplus(i+1) g(n)=(f \oplus i g(n)) \oplus g(n), \\
& f \Theta(i+1) g(n)=(f \Theta i g(n)) \Theta g(n) .
\end{aligned}
$$

Similarly, the multiscale morphological opening, closing, opening-closing, and closing-opening operations of signals are as shown in Equations (11) to (14):

$$
\begin{aligned}
(f \circ g)_{i}(n) & =(f \Theta i g(n)) \oplus i g(n), \\
(f \cdot g)_{i}(n) & =(f \oplus i g(n)) \Theta i g(n), \\
F_{C O}(f(n)) & =(f \cdot i g(n)) \circ i g(n),
\end{aligned}
$$




$$
F_{O C}(f(n))=(f \circ i g(n)) \cdot i g(n) .
$$

2.4. Adaptive Multiscale Combined Morphological Filter. The environment and working conditions of the bearing are often complex and changeable; consequently, noise in the collected signal of the bearing is also complex and diverse; thus, it is difficult to achieve an ideal filtering effect by using a singlescale structuring element. To enable the morphological filter to deal with noise in various situations, multiple groups of structuring elements with different scales are adopted to carry out morphological closing-opening and openingclosing combined filtering for a signal based on the concept of overall multiscale filtering. In addition, structuring elements with different scales are used successively in the same group. The final scale signal is obtained by adaptive weighting combination for each group of operation results. The principle is as follows:

$$
\left\{\begin{aligned}
F_{1 C O}(f(n))= & \left(f \cdot g_{11} \circ g_{12}\right)(n), \\
F_{1 O C}(f(n))= & \left(f \circ g_{11} \cdot g_{12}\right)(n), \\
F_{2 C O}(f(n))= & \left(f \cdot g_{21} \circ g_{22}\right)(n), \\
F_{2 O C}(f(n))= & \left(f \circ g_{21} \cdot g_{22}\right)(n), \\
& \cdots \cdots \\
F_{i C O}(f(n))= & \left(f \cdot g_{i 1} \circ g_{i 2}\right)(n), \\
F_{i O C}(f(n))= & \left(f \circ g_{i 1} \cdot g_{i 2}\right)(n) .
\end{aligned}\right.
$$

In Equation (15), $g_{11} \subseteq g_{12}, g_{21} \subseteq g_{22}$, and $g_{i 1} \subseteq g_{i 2}$.

The final signal filtered by $k$ groups of structuring elements is as shown in Equation (16):

$$
F(f)=\sum_{i=1}^{k}\left(\lambda_{i 1} F_{i C O}(f(n))+\lambda_{i 2} F_{i O C}(f(n))\right)
$$

where $\lambda_{i 1}$ represents the weight of the results of the morphological closing-opening operation on the signal by the $i$ th group of structuring elements in the final signal. $\lambda_{i 2}$ represents the weight of the results of the morphological opening-closing operation on the signal by the $i$ th group of structuring elements in the final signal. $\lambda_{i 1}$ and $\lambda_{i 2}$ can be calculated by Equations (17) to (20):

$$
\begin{aligned}
& \delta_{i 1}=\sum_{n=1}^{N}\left|F_{i C O}(f(n))-f(n)\right|, \\
& \delta_{i 2}=\sum_{n=1}^{N}\left|F_{i O C}(f(n))-f(n)\right|, \\
& \lambda_{i 1}=\frac{\delta_{i 1}}{\sum_{i=1}^{k}\left(\delta_{i 1}+\delta_{i 2}\right)}, \\
& \lambda_{i 2}=\frac{\delta_{i 2}}{\sum_{i=1}^{k}\left(\delta_{i 1}+\delta_{i 2}\right)} .
\end{aligned}
$$

It can be seen that the morphological closing-opening (opening-closing) operation results from large-scale structuring elements have a large proportion of weight in the final signal, and the morphological closing-opening (openingclosing) operation results from small-scale structuring elements have a small proportion of weight, which considers denoising and protecting details under the premise of giving first priority to denoising.

The process of the proposed morphological filter is as follows:

(1) Read the signal $f(n)$ to be processed

(2) Calculate the value of $d$ (the number of sampling points in a pulse period) according to the sampling frequency and pulse frequency (or theoretical fault frequency)

(3) Obtain the width $(0.6 d)$ of the flat structuring element

(4) According to Equation (15), use $2 i$ (the value of $i$ is optional) groups of different scale structuring elements to carry out morphological closing-opening and morphological opening-closing operations for $f_{1}(n)$ and obtain the final signal $f_{1}(n)$ according to Equations (16) to (20)

(5) Implement a fast Fourier transform of $f_{1}(n)$ to obtain its spectrum $F_{1}(n)$

(6) Output $f_{1}(n)$ and $F_{1}(n)$

\section{Analysis of the Filtering Effect for the Simulation Signal}

To test the effect of the proposed adaptive multiscale combined morphological filter, a simulation signal is created for filtering research, which is shown in Equation (21):

$$
x(t)=x_{1}(t)+x_{n}(t) .
$$

In Equation (21), $x_{1}(t)$ is a periodic pulse attenuation signal with a frequency of $60 \mathrm{~Hz}$ and a weekly attenuation function of $e^{-800 \cdot t} \cdot \cos (2 \pi \cdot 800 \cdot t) ; x_{n}(t)$ is a white Gaussian noise signal. The sampling frequency of the signal is $6000 \mathrm{~Hz}$, and the sampling time is $1 \mathrm{~s}$.

The amplitude of the flat structuring element is 0 . According to [19], the width of the structuring element was selected as $0.6 d$ ( $d$ is the number of sampling points in a pulse period). Since the sampling frequency is $6000 \mathrm{~Hz}$ and the pulse frequency is $60 \mathrm{~Hz}$, it can be seen that $d=100$, so the width of the structuring element is 60 .

The waveform and frequency spectrum of the simulated signal are shown in Figure 1. Clearly, there is considerable noise in the simulation signal; the signal-to-noise ratio is low; the pulse signal is completely submerged by noise; the frequency spectrum is relatively messy; neither the waveform nor the frequency spectrum can reflect the periodic characteristics of the pulse signal. 


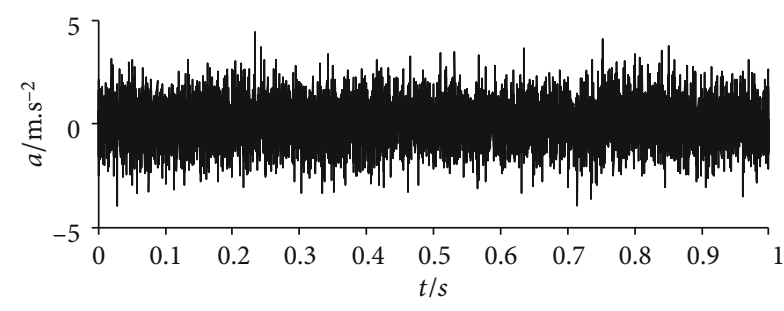

(a)

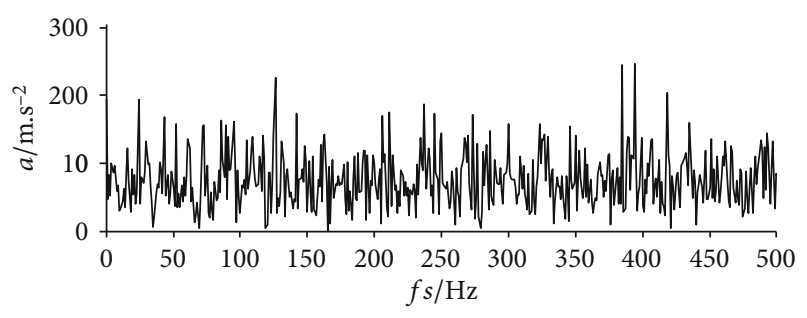

(b)

FIGURE 1: Simulated signal and its frequency spectrum. (a) The waveform of the simulated signal. (b) The frequency spectrum of the simulated signal.

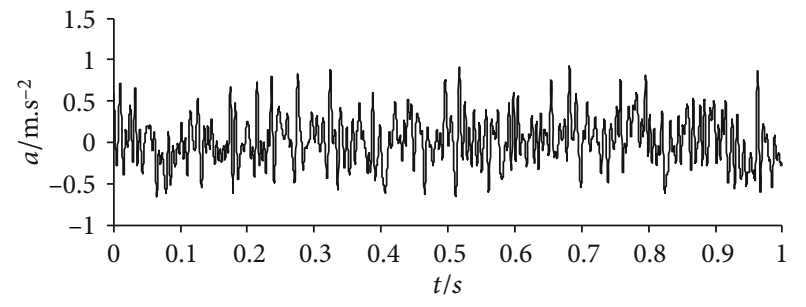

(a)

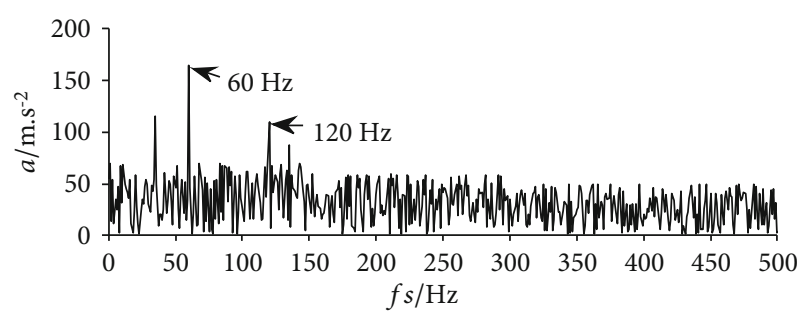

(b)

Figure 2: Waveform and frequency spectrum of the simulated signal denoised by the dual-tree complex wavelet. (a) Waveform. (b) Frequency spectrum.

The waveform and frequency spectrum of the simulated signal denoised by the dual-tree complex wavelet are shown in Figure 2. It can be seen obviously that noise in the signal is suppressed to a certain extent by the dual-tree complex wavelet, but considerable noise still remains. The characteristic frequency of the pulse signal can be reflected in the frequency spectrum, but there is still considerable interference. Moreover, the periodic characteristic of the signal is not obvious.

The waveform and frequency spectrum of the simulated signal denoised by the traditional morphological filter with small-scale structuring elements are shown in Figure 3. It can be seen obviously that the denoising effect is better than

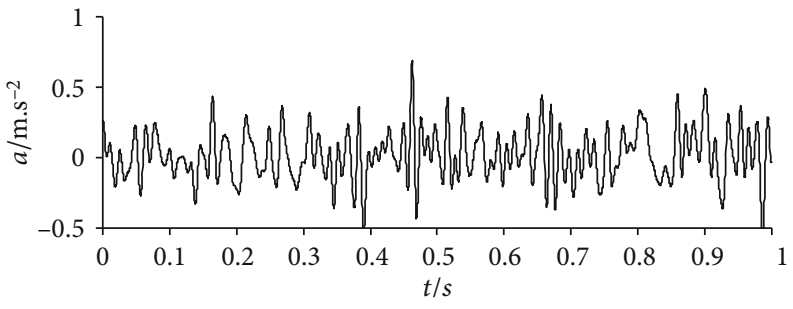

(a)

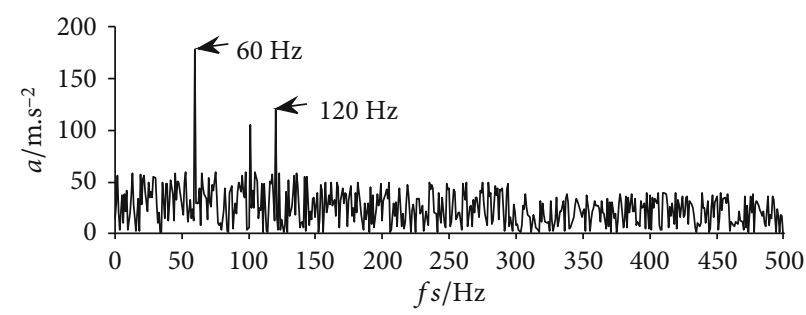

(b)

FIGURE 3: Waveform and frequency spectrum of simulated signal denoised by traditional morphological filter with small-scale structuring elements. (a) Waveform. (b) Frequency spectrum.

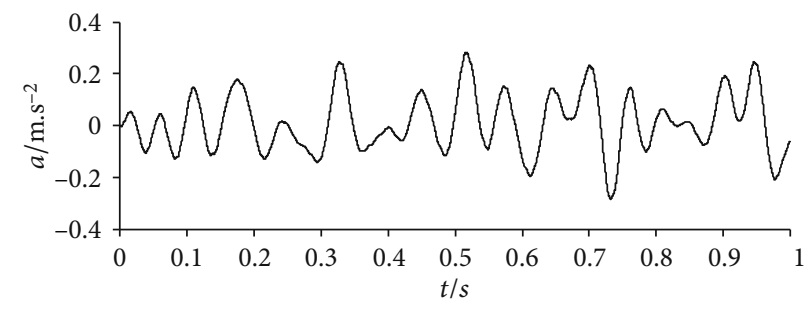

(a)

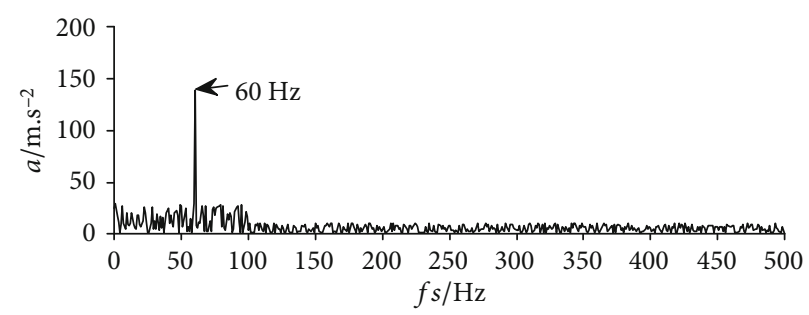

(b)

FIgURE 4: Waveform and frequency spectrum of simulated signal denoised by traditional morphological filter with large-scale structuring elements. (a) Waveform. (b) Frequency spectrum.

that of the dual-tree complex wavelet, but a certain amount of noise remains. The characteristic frequency of the pulse signal can be reflected in the frequency spectrum, but the periodic characteristic of the signal is still not sufficiently obvious because of the interference of the left noise.

The waveform and frequency spectrum of the simulated signal denoised by the traditional morphological filter with large-scale structuring elements are shown in Figure 4. It can be seen obviously that some useful signals in the waveform are filtered, resulting in distortion of the signal, and the frequency spectrum only reflects the primary frequency of the pulse signal. 


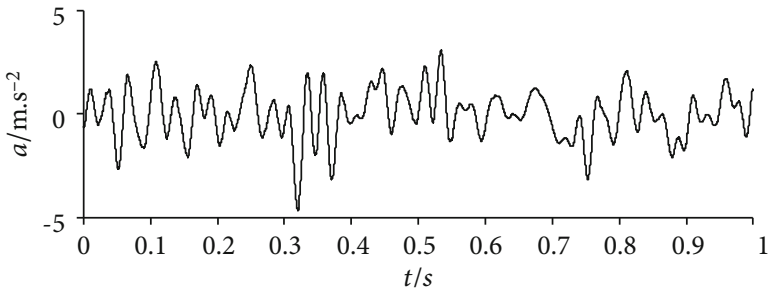

(a)

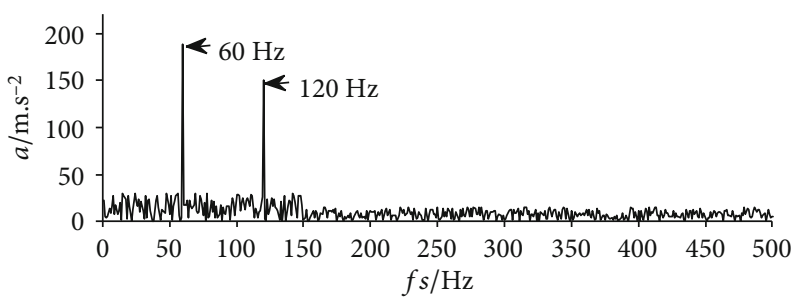

(b)

FIGURE 5: Waveform and frequency spectrum of the simulated signal denoised by the adaptive multiscale combined morphological filter. (a) Waveform. (b) Frequency spectrum.

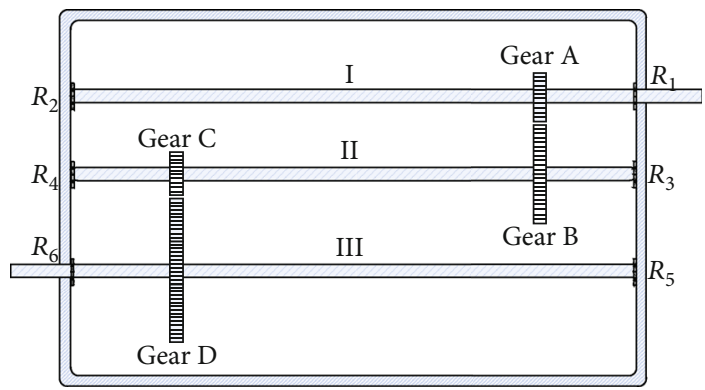

FIgURE 6: Structure diagram of the tested gearbox.

The waveform and frequency spectrum of the simulated signal denoised by the adaptive multiscale combined morphological filter are shown in Figure 5. It can be seen obviously that not only a great quantity of noise in the signal is effectively filtered but also the distortion of the effective signal is avoided. Only a miniscule quantity of clutter remains in the frequency spectrum, which reflects both the primary and double frequency of the pulse signal. The denoising effect is obviously better than that of the dual-tree complex wavelet and traditional morphological filter.

\section{Analysis of the Filtering Effect for the Bearing Fault Signal}

The bearing fault signal is collected through an experiment involving a two-stage transmission gearbox whose structure is shown in Figure 6. In the first-stage transmission, gear A on the input shaft (I) and gear B on the intermediate shaft (II) mesh with each other. The tooth numbers of gear $\mathrm{A}$ and gear B are 23 and 47 , respectively. In the second-stage transmission, gear $\mathrm{C}$ on the intermediate shaft (II) and gear $\mathrm{D}$ on the output shaft (III) mesh with each other. The tooth numbers of gear $\mathrm{C}$ and gear $\mathrm{D}$ are 19 and 78 , respectively.

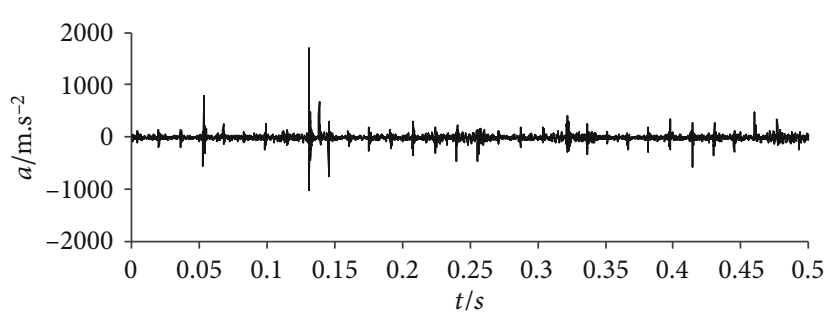

(a)

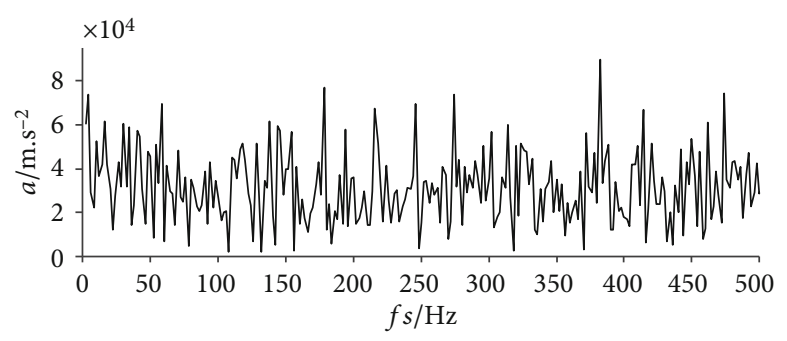

(b)

FIgURE 7: Waveform and frequency spectrum of bearing $R_{3}$ with an outer ring defect. (a) Waveform. (b) Frequency spectrum.

During the experiment, the outer ring of bearing $R_{3}$ on the intermediate shaft (II) is set to be defective, and the acceleration sensor is set at the bearing block of $R_{3}$ to collect the fault signal. The speed of the input shaft (I) is $1520 \mathrm{r} / \mathrm{min}$, the sampling frequency is $10 \mathrm{kHz}$, and the number of sampling points is 5000. It can be calculated that the characteristic frequency of the bearing $R_{3}$ outer ring defect signal is $126.4 \mathrm{~Hz}$.

The width of the structuring element is chosen as $0.6 d$ ( $d$ is the number of sampling points in a pulse period). Since the sampling frequency is $10 \mathrm{kHz}$ and the pulse frequency is $126.4 \mathrm{~Hz}$, it can be seen that $d=79$, so the width of the structuring element is 47 .

The waveform and frequency spectrum of bearing $R_{3}$ with an outer ring defect obtained by the experiment are shown in Figure 7.

The waveform and frequency spectrum of bearing $R_{3}$ with an outer ring defect signal denoised by a dual-tree complex wavelet are shown in Figure 8. It can be clearly seen that the dual-tree complex wavelet can filter out most of the noise in the signal, but the filtering is insufficient. The frequency spectrum reflects the primary frequency $(126 \mathrm{~Hz})$ and double frequency $(252 \mathrm{~Hz})$ of the pulse signal; however, there is excessive clutter in the frequency spectrum, which easily produces interference.

The waveform and frequency spectrum of bearing $R_{3}$ with an outer ring defect signal denoised by a traditional morphological filter with small-scale structuring elements are shown in Figure 9. It can be seen obviously that the noise is less than that by the dual-tree complex wavelet; the frequency spectrum obviously highlights the primary frequency $(126 \mathrm{~Hz})$ and double frequency $(252 \mathrm{~Hz})$ of the pulse signal, and the clutter is further reduced as well.

The waveform and frequency spectrum of bearing $R_{3}$ with an outer ring defect signal denoised by a traditional morphological filter with large-scale structuring elements 


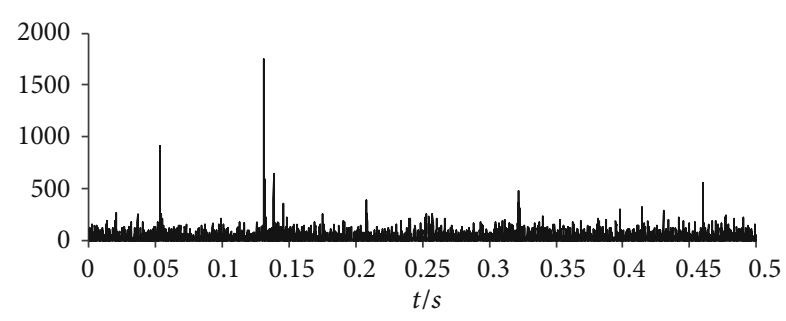

(a)

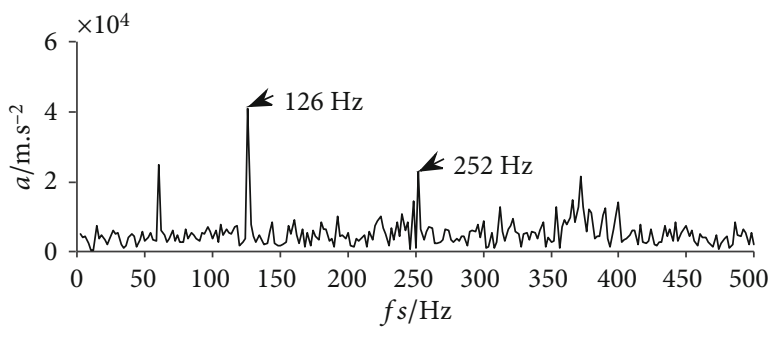

(b)

FIGURE 8: Waveform and frequency spectrum of bearing $R_{3}$ with an outer ring defect signal denoised by a dual-tree complex wavelet. (a) Waveform. (b) Frequency spectrum.

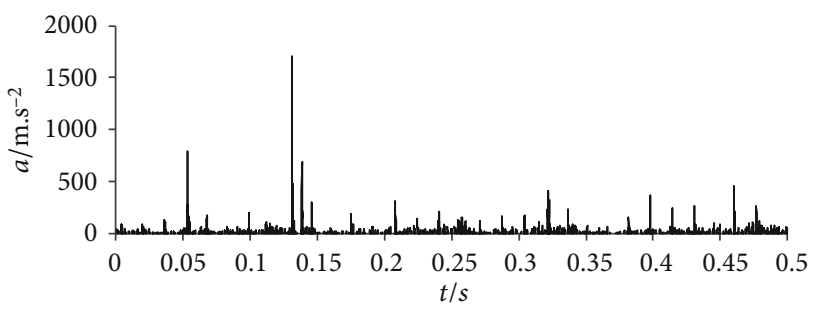

(a)

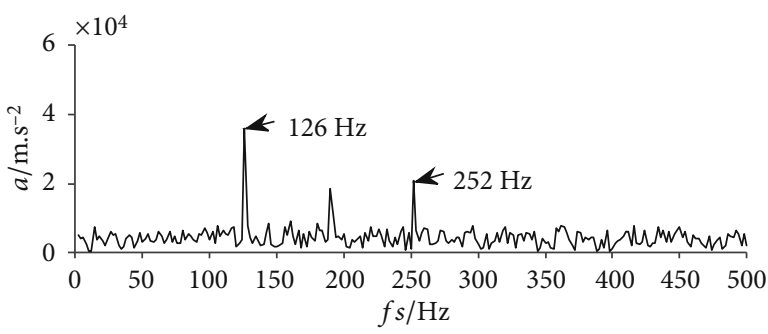

(b)

FIGURE 9: Waveform and frequency spectrum of bearing $R_{3}$ with an outer ring defect signal denoised by a traditional morphological filter with small-scale structuring elements. (a) Waveform. (b) Frequency spectrum.

are shown in Figure 10. It can be seen obviously that the signal is distorted, with a loss of partial effective information; the frequency spectrum only reflects the primary frequency $(126 \mathrm{~Hz})$ of the pulse signal.

The waveform and frequency spectrum of bearing $R_{3}$ with an outer ring defect signal denoised by an adaptive multiscale combined morphological filter are shown in Figure 11. It can be seen obviously that the signal details are well preserved while the noise is effectively filtered out; the frequency spectrum clearly reflects the primary frequency $(126 \mathrm{~Hz})$ and

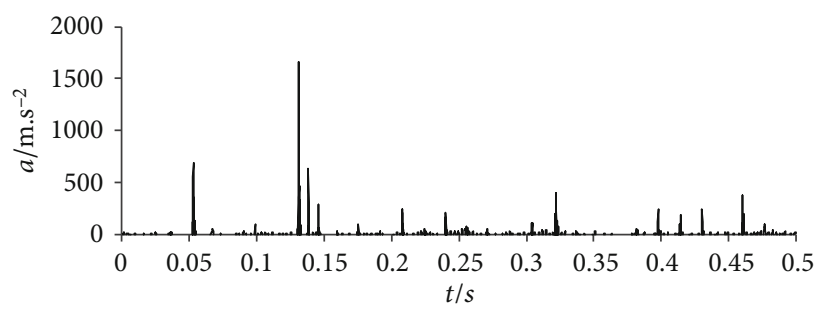

(a)

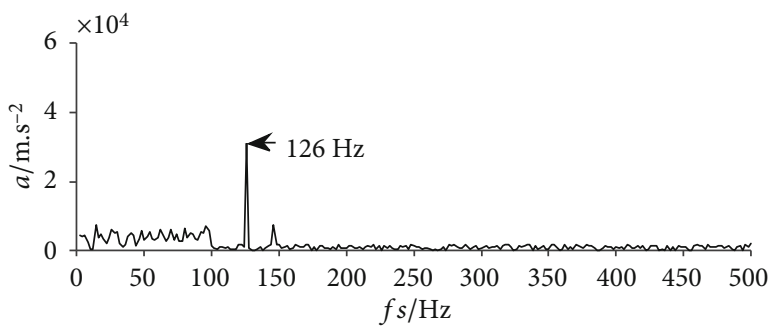

(b)

FIgURE 10: Waveform and frequency spectrum of bearing $R_{3}$ with an outer ring defect signal denoised by a traditional morphological filter with large-scale structuring elements. (a) Waveform. (b) Frequency spectrum.

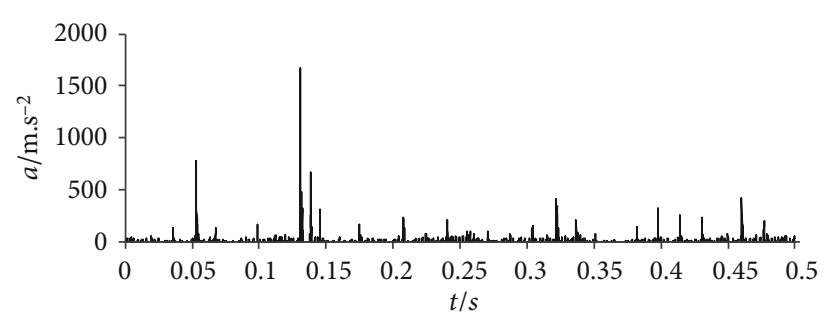

(a)

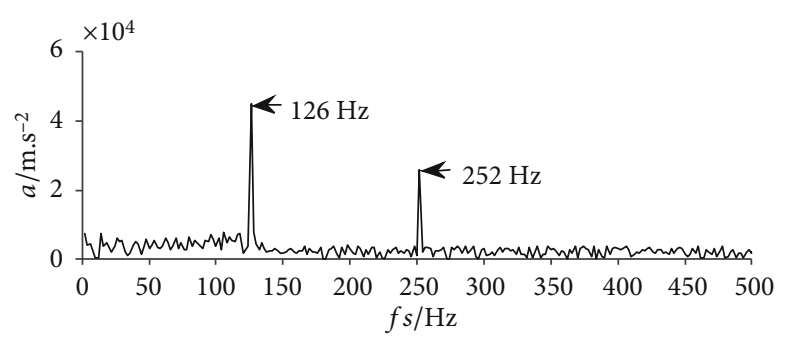

(b)

FIgURE 11: Waveform and frequency spectrum of bearing $R_{3}$ with an outer ring defect signal denoised by an adaptive multiscale combined morphological filter. (a) Waveform. (b) Frequency spectrum.

double frequency $(252 \mathrm{~Hz})$ of the pulse signal; furthermore, the clutter is extremely small.

In recent years, Qin et al. [20] proposed an adaptive singular value decomposition method (ASVD) for gearbox fault signal filtering. Cui et al. [21] proposed an improved switching Kalman filter (ISKF) to filter bearing fault signals. We use these two methods to denoise the outer ring defect signal of bearing $R_{3}$, and the results are shown in Figures 12 and 13. It can be seen obviously that although these two methods can filter out most of the noise in the signal, yet they are both 


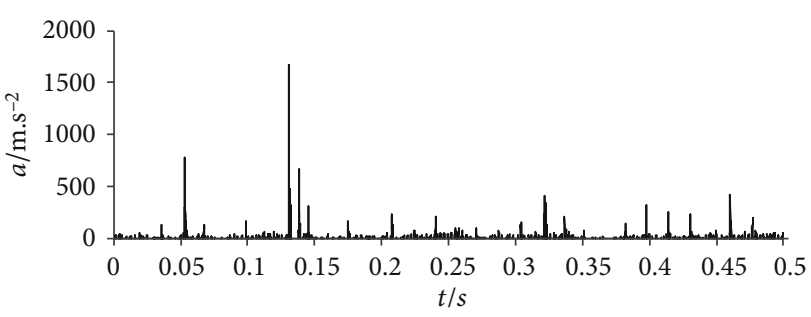

(a)

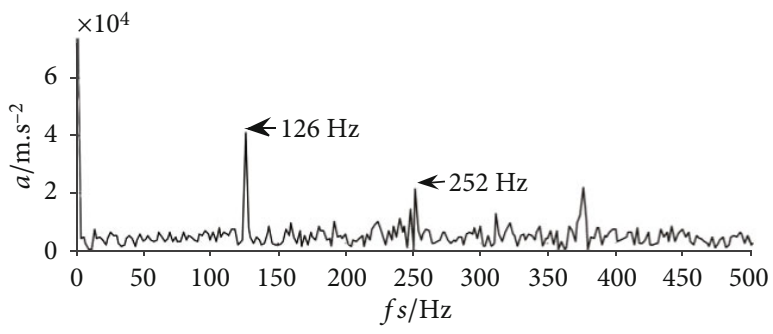

(b)

FIGURE 12: Waveform and frequency spectrum of bearing $R_{3}$ with an outer ring defect signal denoised by ASVD. (a) Waveform. (b) Frequency spectrum.

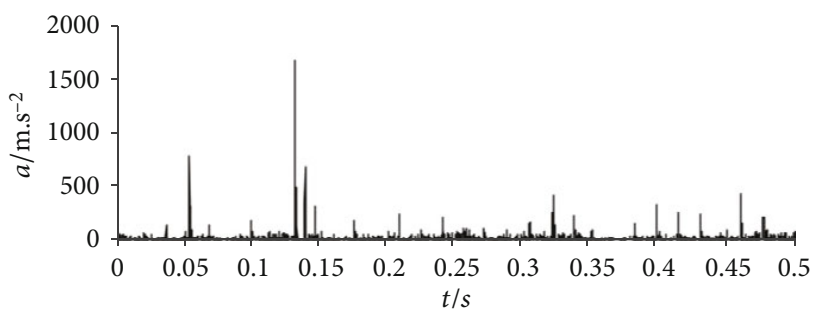

(a)

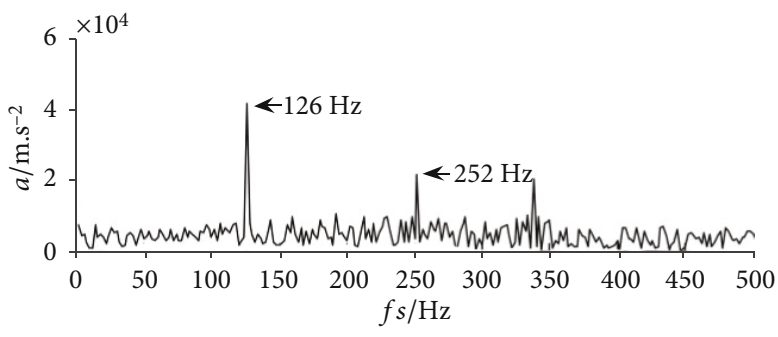

(b)

FIGURE 13: Waveform and frequency spectrum of bearing $R_{3}$ with an outer ring defect signal denoised by ISKF. (a) Waveform. (b) Frequency spectrum.

inferior to our proposed filter. The frequency spectrum reflects the primary frequency $(126 \mathrm{~Hz})$ and double frequency $(252 \mathrm{~Hz})$ of the pulse signal, but there is still much interference in the high-frequency part. By comparing with these two methods, the novelty of our proposed adaptive multiscale combined morphological filter is reflected.

\section{Conclusions}

To address the insufficient denoising effects of traditional filters, an adaptive multiscale combined morphological fil- ter is proposed, which is composed of morphological closing-opening and opening-closing operations. The advantages of the proposed filter are verified by simulations and experiments, as specifically manifested in the following three aspects:

(1) The morphological filter proposed in this paper uses multiple groups of structuring elements with different scales to carry out morphological closingopening and opening-closing combined filtering for signals. In addition, structuring elements with different scales are used successively in the same group. The final scale signal is obtained by a self-adaptive weighting combination of each group of operation results. The morphological closing-opening (opening-closing) operation results by large-scale structuring elements have a large proportion of weight in the final signal, and the morphological closing-opening (opening-closing) operation results by small-scale structuring elements have a small proportion of weight, which can take into account the denoising and protecting details of the signal under the premise of giving first priority to denoising

(2) The proposed filter can filter out noise more thoroughly and extract pulse signals more effectively than dual-tree complex wavelets, ASVD and ISKF. Compared with the traditional morphological filter with small-scale structuring elements, which has an incomplete denoising effect, the proposed filter can filter out noise more effectively; compared with the traditional morphological filter with large-scale structuring elements, which loses part of the useful signal, the proposed filter can preserve the useful signal much better

(3) The proposed filter takes into account both positive and negative pulses in the signal; therefore, it has strong adaptability to complex and variable noise in actual environments. Thus, the filter provides a new effective method for rotating machinery fault diagnosis

\section{Data Availability}

The data used to support this research can be obtained from the corresponding author on request.

\section{Conflicts of Interest}

The authors declare that there is no conflict of interest regarding the publication of this paper.

\section{Acknowledgments}

The research has been supported by the National Natural Science Foundation of China (grant number 51305454). 


\section{References}

[1] I. Bloch, "Duality vs. adjunction for fuzzy mathematical morphology and general form of fuzzy erosions and dilations," Fuzzy Sets and Systems, vol. 160, no. 13, pp. 1858-1867, 2009.

[2] H. Chen, B. Jiang, N. Lu, and Z. Mao, "Deep PCA based realtime incipient fault detection and diagnosis methodology for electrical drive in high-speed trains," IEEE Transactions on Vehicular Technology, vol. 67, no. 6, pp. 4819-4830, 2018.

[3] X. Gu and C. Chen, "Rolling bearing fault signal extraction based on stochastic resonance-based denoising and VMD," International Journal of Rotating Machinery, vol. 2017, Article ID 3595871, 12 pages, 2017.

[4] R. M. Haralick, S. R. Sternberg, and X. Zhuang, "Image analysis using mathematical morphology," IEEE Transactions on Pattern Analysis and Machine Intelligence, vol. 9, no. 4, pp. 532-550, 1987.

[5] P. H. Rodríguez, J. B. Alonso, M. A. Ferrer, and C. M. Travieso, "Application of the Teager-Kaiser energy operator in bearing fault diagnosis," ISA Transactions, vol. 52, no. 2, pp. 278284, 2013.

[6] P. Jayaswal, A. K. Wadhwani, and K. B. Mulchandani, "Machine fault signature analysis," International Journal of Rotating Machinery, vol. 2008, Article ID 583982, 10 pages, 2008.

[7] C. Li, M. Liang, Y. Zhang, and S. Hou, "Multi-scale autocorrelation via morphological wavelet slices for rolling element bearing fault diagnosis," Mechanical Systems and Signal Processing, vol. 31, pp. 428-446, 2012.

[8] C. Li and M. Liang, "Continuous-scale mathematical morphology-based optimal scale band demodulation of impulsive feature for bearing defect diagnosis," Journal of Sound and Vibration, vol. 331, no. 26, pp. 5864-5879, 2012.

[9] F. Namdari and M. Salehi, "High-speed protection scheme based on initial current traveling wave for transmission lines employing mathematical morphology," IEEE Transactions on Power Delivery, vol. 32, no. 1, pp. 246-253, 2017.

[10] R. A. Peters, “A new algorithm for image noise reduction using mathematical morphology," IEEE Transactions on Image Processing, vol. 4, no. 5, pp. 554-568, 1995.

[11] A. S. Raj and N. Murali, "Early classification of bearing faults using morphological operators and fuzzy inference," IEEE Transactions on Industrial Electronics, vol. 60, no. 2, pp. 567574, 2013.

[12] F. Y. Shih and S. Cheng, "Adaptive mathematical morphology for edge linking," Information Sciences, vol. 167, no. 1-4, pp. 921, 2004.

[13] J. Shuai, C. Shen, and Z. Zhu, "Adaptive morphological feature extraction and support vector regressive classification for bearing fault diagnosis," International Journal of Rotating Machinery, vol. 2017, Article ID 2384184, 10 pages, 2017.

[14] Y. Wei and L. Pei, "Detection of power quality disturbances based on mathematical morphology (MM) filter," Automation of Electric Power Systems, vol. 4, no. 10, pp. 13-17, 2002.

[15] X. Gong and W. Qiao, "Bearing fault diagnosis for direct-drive wind turbines via current-demodulated signals," IEEE Transactions on Industrial Electronics, vol. 60, no. 8, pp. 34193428, 2013.

[16] H. Yu, H.-r. Li, Z.-k. Tian, and W.-g. Wang, "Rolling bearing degradation state identification based on LPP optimized by GA," International Journal of Rotating Machinery, vol. 2016, Article ID 9281098, 10 pages, 2016.
[17] J. Zhang, H. Yao, and G. Rizzoni, "Fault diagnosis for electric drive systems of electrified vehicles based on structural analysis," IEEE Transactions on Vehicular Technology, vol. 66, no. 2, pp. 1027-1039, 2017.

[18] L. Zhang, J. Xu, J. Yang, D. Yang, and D. Wang, "Multiscale morphology analysis and its application to fault diagnosis," Mechanical Systems and Signal Processing, vol. 22, no. 3, pp. 597-610, 2008.

[19] N. G. Nikolaou and I. A. Antoniadis, "Application of morphological operators as envelope extractors for impulsive- type periodic signals," Mechanical Systems and Signal Processing, vol. 17, no. 6, pp. 1147-1162, 2003.

[20] Y. Qin, Q. L. Zhang, and Y. Zhao, "Fault diagnosis method for planetary gearboxes based on adaptive SVD," Journal of Vibration and Shock, vol. 37, no. 17, pp. 122-127, 2018.

[21] L. CUI, W. Xin, W. Huaqing, X. Yonggang, and Z. Jianyu, "Feature extraction of bearing fault based on improved switching Kalman filter," Journal of Mechanical Engineering, vol. 55, no. 7, pp. 44-51, 2019. 